\title{
L'HYBRIDATION NATURELLE ENTRE LE BARBEAU COMMUN ET LE BARBEAU MERIDIONAL EN FRANCE : COMPTE RENDU DE DIX ANNÉES DE RECHERCHE.
}

\section{CRESPIN et P. BERREBI}

Laboratoire Génome et Populations, Université Montpellier II, CC 063, Place Eugène Bataillon, 34095 Montpellier Cedex 05, France.

\section{RÉSUMÉ}

Les deux espèces de barbeaux de France s'hybrident spontanément dans plusieurs bassins. Les modalités de cette hybridation ont été recherchées au travers de nombreuses études relevant de disciplines différentes (biogéographie, éthologie, génétique et parasitologie). Cet article se veut une synthèse des différents travaux consacrés à cette hybridation en même temps qu'une caractérisation de l'état de l'hybridation dans toute la France.

Mots-clés : Barbus, hybridation, zone hybride, génétique des populations, allozymes.

\section{NATURAL HYBRIDIZATION BETWEEN THE COMMON BARBEL AND THE MEDITERRANEAN BARBEL IN FRANCE : REPORT OF TEN YEARS OF RESEARCH.}

\begin{abstract}
The aim of this publication is double : one is to gather all the different studies concerning the hybridization between the common barbel and the Mediterranean barbel for setting off the mechanisms of this hybridization ; second is to collect from different searchers unpublished data about these two species in order to have an idea of the extent of this hybridization in France.These two species, Barbus barbus and Barbus meridionalis, are quite different morphologically (figure 1) and ecologically. The former lives in large rivers with a strong current whereas the second one can live in small mountain tributaries.
\end{abstract}

In southern France, hybridization occurs in few rivers. The sampled fish were analysed by electrophoresis on horizontal starch gels at ten diagnostic loci. A genetic hybrid index (Gl) corresponding conventionally to the percentage of meridionalis genes in an individual or a population was calculated.

A hybrid zone has been identified in the Lergue river, near Montpellier, by the presence of enzymatic clines (figure 3). The presence of sharp enzymatic clines and linkage disequilibrium indicate that this hybrid zone is a tension zone, in which a balance between selection and dispersal maintains the clines. Parasitical studies, using a natural specific parasite of Barbus meridionalis as genetic marker, have demonstrated the importance of the behaviour of fish for being infested or not, and so, for hybrid zone stability.

Other studies in natura or in aquaria have shown too the existence of ecological differences between the two species and their hybrids. Paleontological evidences suggest that the two species only came into contact since last glaciation, date of the arrival from the nearest catchment area of Barbus barbus in Hérault basin. 
A map showing the extent of hybridization between the two species in France is given (figure 2). All these results are used for trying to understand how this hybridization is functioning.

Key-words : Barbus, hybridization, hybrid zone, population genetics, allozymes, parasitology.

\section{INTRODUCTION}

Du point de vue évolutif, on peut distinguer deux types d'hybridations. Le premier est celui qui produit des hybrides totalement stériles (BERREBI et al., 1989 ; KONKLE et PHILIPP, 1992) ; ceux-ci n'auront tout au plus qu'une influence dans l'écologie du milieu fréquenté. Le deuxième type d'hybridation produit des descendants fertiles, plus ou moins complètement. Dans ce cas-là, par backcrosses avec les espèces parentales, il peut y avoir interpénétration entre espèces (introgression) et donc modification des deux génomes parentaux au cours des générations. Il semblerait qu'une espèce de Catostomidés américains ait pris naissance de la sorte en étant le résultat de la fusion de deux espèces préexistantes (MENZEL, 1976). L'importance évolutive, au moins sur le plan qualitatif, de ce phénomène est évidente.

De nombreuses hybridations ont été décrites chez les Poissons. Du fait des modalités de leur fécondation externe (libération des gamètes dans un milieu dynamique), les barrières pré-zygotiques semblent plus facilement franchissables que chez les Tétrapodes. Les hybridations inter-spécifiques sont relativement courantes (ECONOMIDIS et SINIS, 1988 ; CAMPTON, 1987 et 1990 ; VERSPOOR et HAMMAR, 1991) et on connaît même des cas, encore nombreux, d'hybridations inter-génériques (BERREBI et al., 1989).

Le cas de l'hybridation entre le barbeau commun (Barbus barbus Linné 1758) et le barbeau méridional (Barbus meridionalis Risso 1826), signalé pour la première fois en 1987 (BERREBI et al., 1987), parce qu'il produit des hybrides fertiles et parce que l'on en connaît plusieurs cas similaires géographiquement isolées, est particulièrement intéressant. Le texte de synthèse qui suit a pour but de faire le point sur les connaissances de cette hybridation qui a suscité de nombreux travaux.

\section{LES ESPECES}

Les deux espèces qui nous intéressent sont des Cyprinidae d'eau douce tétraploïdes. Elles possèdent 100 chromosomes alors que la plupart des espèces de cette famille en ont de 48 à 52 (HAFEZ, 1981). Ces deux espèces sont bien différentes tant sur le plan écologique que morphologique.

\section{Morphologie}

Les traits morphologiques distinguant ces deux espèces sont faciles à reconnaître. On a même démontré que cinq variables morphologiques suffisaient à décrire leurs différences (BERREBI et al., 1993).

- Le barbeau méridional est plus trapu que le barbeau commun alors que ce dernier est de taille nettement supérieure (tailles maximums : $30 \mathrm{~cm}$ et $1 \mathrm{~m}$ ).

— Le barbeau méridional a entre 45 et 55 écailles sur la ligne latérale (55 à 65 chez le barbeau commun).

- Le barbeau méridional a une nageoire anale longue qui atteint la base de la nageoire caudale, l'autre espèce a une nageoire anale courte.

- Le barbeau commun a une nageoire dorsale triangulaire concave, dont le dernier rayon dur, fortement ossifié, porte des denticules au côté postérieur, le méridional n'en a pas et sa nageoire dorsale est convexe et relativement molle.

Les hybrides possèdent des caractères intermédiaires entre les espèces parentales (figure 1) 


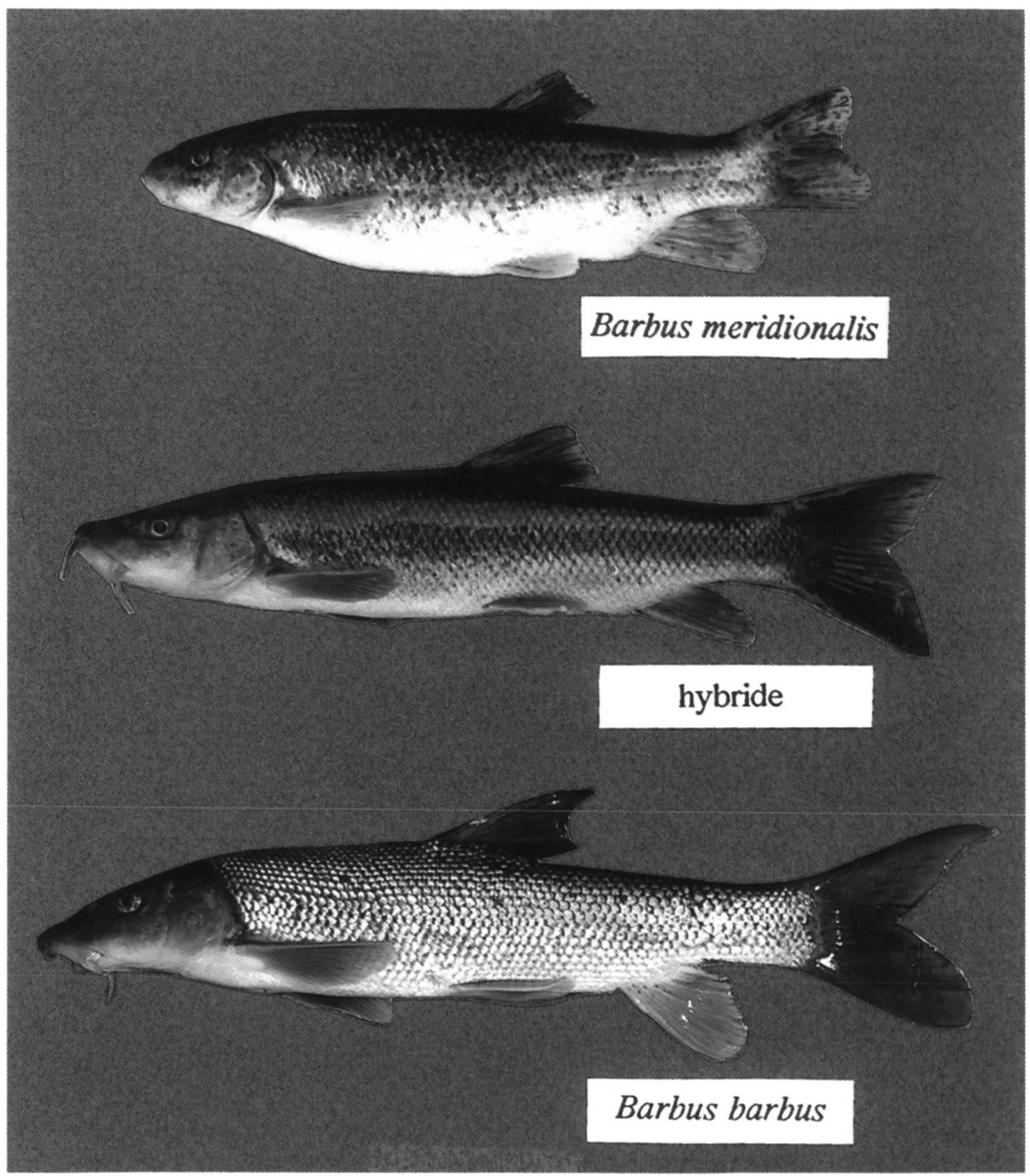

Figure 1 : Aspect des deux espèces de barbeaux : remarquer l'hydrodynamisme de la forme du corps ; la couleur avec ou sans taches; la forme des nageoires arrondies ou pointues; la longueur de la nageoire anale atteignant ou pas la base de la nageoire caudale.

Figure 1 : Morphology of the two barbels : notice the hydrodynamism of the body ; the color, marbled or not ; the shape of the fins, rounded or pointed ; the length of the anal fin, whether it reaches the beginning of the caudal fin. 


\section{Ecologie}

Les caractéristiques écologiques, quoique peu étudiées, apparaissent aussi assez différentes. Le barbeau commun affectionne les cours d'eau de plaine au débit important, bien oxygéné. Le barbeau méridional semble être davantage une espèce de semimontagne (entre 200 et $1000 \mathrm{~m}$ selon BERREBI et al., 1988), capable de vivre dans des torrents susceptibles de s'assécher en été en région méditerranéenne. Dans les rivières où les deux espèces sont présentes, on retrouve le barbeau commun à l'aval, dans les cours inférieur et moyen, et le barbeau méridional à l'amont, dans le cours supérieur. Cette répartition altitudinale contrastée a été attribuée à l'existence d'une compétition interspécifique entre les deux taxons (BERREBI et al., 1988) : le barbeau commun repousserait le barbeau méridional dans la partie supérieure des cours d'eau. Les conditions drastiques qui règnent dans cette partie (faible à très faible débit d'eau, assèchement en été, eutrophisation...) ne pourraient être tolérées par le barbeau commun. Le barbeau méridional du fait de sa résistance à ces mêmes conditions peut se maintenir très en amont. Cette hypothèse est corroborée par le fait que les barbeaux méridionaux vivant dans une rivière des Pyrénées-Orientales, le Tech, se rencontrent en plaine. Or, dans cette région, le barbeau commun est absent, pour des raisons, sans doute, paléohistoriques (PERSAT et BERREBI, 1990). En fait, aucune étude écologique n'a été consacrée à la recherche de la compétition entre les deux espèces et si celle-ci existe, on ne sait pas sur quelles bases elle opère. La compétition est-elle indirecte pour l'appropriation d'une ressource (nourriture ou frayère) ou est-elle directe par agression entre les représentants des deux espèces (prédation des alevins et/ou des oeufs) ? Ajoutons que, quoi qu'il en soit, la compétition reste un phénomène très difficile à mettre en évidence. Par exemple, on peut imaginer que la répartition altitudinale actuelle des barbeaux est la conséquence d'une compétition passée qui n'existe plus actuellement. $\mathrm{Ce}$ que nous observons aujoud'hui n'est plus alors que le fantôme de la compétition (“competition ghost») ancienne (BLONDEL, 1986). Selon cette hypothèse, on ne retrouvera donc aucune preuve d'une compétition actuellement.

\section{Origine des peuplements}

Le barbeau méridional est implanté depuis fort longtemps dans le sud de la France. Une forme ancestrale y est connue, à l'état fossile, datant d'environ cinq millions d'années (miocène supérieur). Le barbeau commun est originaire d'Europe centrale et serait arrivé “récemment» en Europe occidentale : depuis le pliocène, il y a deux millions d'années, et sur la côte méditerranéenne : probablement depuis les dernières glaciations, il y a 10 ou 15000 ans seulement (PERSAT et BERREBI, 1990). Certaines régions n'ont pas été atteintes par le barbeau commun comme le Roussillon, à l'Est des Pyrénées, ou l'extrême Bretagne.

\section{EXTENSION DE L'HYBRIDATION EN FRANCE}

A l'échelle de la France, seuls quelques bassins hydrographiques, sur la côte méditerranéenne, abritent simultanément les deux espèces. Ces cours d'eau ont pour la plupart été échantillonnés. Mille soixante cinq poissons ont été analysés entre 1983 et 1993 et seule une partie des résultats a été publiée. Nous nous proposons de récapituler l'ensemble des résultats obtenus. Le tableau I présente le résultat de l'analyse des 49 échantillons effectués sur près de dix ans et la figure 2 les localise. Ceux qui ont donné lieu à des publications sont indiqués. Les autres constituent des données originales.

Deux cas particuliers sont à relever:

- d'une part, celui de la Mare (affluent de l'Orb) qui semble être le résultat de la rupture d'un barrage du 17 e siècle au début des années 80 . A partir de cette date, les riverains assurent avoir vu remonter des gros barbeaux (des barbeaux présumés communs). Une étude ponctuelle a révélé une introgression de $9 \%$ à l'aval de l'emplacement du barrage et de $37 \%$ à l'amont de celui-ci. On peut se demander comment se fait la transition entre ces deux points extrêmes ? Faut-il y voir une zone hybride en formation? 
Tableau I : Récapitulatif des 1065 barbeaux analysés entre mai 1983 et février 1992. ${ }^{\star}=$ données non encore publiées ; ${ }^{\star \star}=$ données non enregistrées.

1 = Berrebi (1984) ; 2 = Berrebi et al. (1988) ; 3 = Le Brun (1988) ; 4 = Persat \& Berrebi (1990); $5=$ Pouyaud (1990) ; $6=$ Berrebi (1991) ; $7=$ Crespin (1992) ; 8 = Le Brun et al. (1992) ; 9 = Berrebi et al. (1993) ; $10=$ Berrebi (en prép.).

Table I : Recapitulation of the analyses of 1065 barbels (may 1983 - february 1992). ${ }^{\star}=$ unpublished data ; ${ }^{\star \star}=$ unrecorded data.

\begin{tabular}{|c|c|c|c|c|c|c|c|}
\hline Date & Station & Rivière & Affluent de & Bassin & $\mathbf{N}$ & GI & Bibliogr. \\
\hline mai 83 & Terrades & Terrades & Gard & Rhône & 36 & 100 & $1,2,4,6$ \\
\hline mai 83 & Pradet & Pradet & Gard & Rhône & 36 & 100 & $1,2,4$ \\
\hline mai 83 & Rieutord & Rieutord & Hérault & Hérault & 36 & 100 & $1,2,4,6$ \\
\hline juin 83 & Amélie/Bains & Tech & Tech & Tech & 36 & 100 & $1,2,4,6$ \\
\hline juin 83 & $\star \star$ & Estéron & Var & Var & 36 & 100 & $1,2,4,6$ \\
\hline juin 84 & St Fréchoux & Lergue & Hérault & Hérault & 11 & 40 & $\star$ \\
\hline sept. 84 & aval & Ouvèze & Rhône & Rhône & 24 & 9 & * \\
\hline sept. 84 & amont & Herbasse & Isère & Rhône & 19 & 100 & 6 \\
\hline sept. 84 & aval & Herbasse & Isère & Rhône & 13 & 5 & 6,9 \\
\hline oct. 84 & St Fréchoux & Lergue & Hérault & Hérault & 11 & 39 & 3,8 \\
\hline nov. 84 & Clermont/H & Dourbie & Hérault & Hérault & 29 & 99 & $3,6,8$ \\
\hline fev. 85 & Bugey & Rhône & Rhône & Rhône & 9 & 0 & 6,9 \\
\hline fev. 85 & $\star \star \star \pi$ & Boyne & Hérault & Hérault & 7 & 100 & $3,6,8$ \\
\hline mars 85 & St Fréchoux & Lergue & Hérault & Hérault & 11 & 49 & 3,8 \\
\hline mars 85 & St Bauzille & Hérault & Hérault & Hérault & 18 & 16 & 3,8 \\
\hline juin 85 & St Fréchoux & Lergue & Hérault & Hérault & 10 & 30 & 3,8 \\
\hline juill. 85 & $\star \star \star$ & Dourbie & Hérault & Hérault & 12 & 99 & $3,6,8$ \\
\hline mars 86 & Canet & Lergue & Hérault & Hérault & 23 & 16 & 3,8 \\
\hline mai 86 & St Fréchoux & Lergue & Hérault & Hérault & 27 & 44 & $\star$ \\
\hline juil. 86 & Le Touch & Louge & Garonne & Garonne & 11 & 84 & * \\
\hline aout 86 & $\star \star$ & Lot & Garonne & Garonne & 8 & 0 & 9 \\
\hline sept. 86 & Rodez & Aveyron & Garonne & Garonne & 7 & 4 & $\star$ \\
\hline sept 86 & Camares & Dourdou & Tarn & Garonne & 7 & 0 & * \\
\hline déc. 86 & St Fréchoux & Lergue & Hérault & Hérault & 17 & 40 & * \\
\hline juil. 87 & $\star \star$ & Margueritte & Lergue & Hérault & 13 & 100 & $3,6,8$ \\
\hline juil. 88 & $* *$ & Ste Croix & Gard & Rhône & 16 & 100 & 6 \\
\hline juil. 88 & ** & Estéron & Var & Var & 23 & 100 & 6 \\
\hline sept. 88 & ** & Aude & Aude & Aude & 9 & 0 & 9 \\
\hline jan. 89 & St André/S & Lergue & Hérault & Hérault & 25 & 15 & 10 \\
\hline jan. 89 & Ceyras & Lergue & Hérault & Hérault & 20 & 16 & 10 \\
\hline jan. 89 & St Fréchoux & Lergue & Hérault & Hérault & 23 & 33 & 10 \\
\hline fev. 89 & C/Aubaygue & Lergue & Hérault & Hérault & 9 & 93 & 6,10 \\
\hline fev. 89 & Fozières & Fozières & Lergue & Hérault & 21 & 100 & 6,10 \\
\hline mars 89 & Rabieux & Lergue & Hérault & Hérault & 14 & 23 & 10 \\
\hline fev. 90 & Puech & Aubaygue & Lergue & Hérault & 8 & 100 & 9 \\
\hline mars 90 & $\star \star$ & Margueritte & Lergue & Hérault & 10 & 99 & 6 \\
\hline avr. 90 & St Fréchoux & Lergue & Hérault & Hérault & 109 & 34 & 5,9 \\
\hline avr. 90 & Puech & Aubaygue & Lergue & Hérault & 7 & 100 & 6,9 \\
\hline juin 90 & Villemagne & Mare & Orb & Orb & 31 & 9 & $\star$ \\
\hline juin 90 & St Gervais & Mare & Orb & Orb & 22 & 34 & $\star$ \\
\hline fev. 92 & St André/S & Lergue & Hérault & Hérault & 25 & 15 & 7 \\
\hline fev. 92 & Ceyras & Lergue & Hérault & Hérault & 30 & 16 & 7 \\
\hline fev. 92 & Rabieux & Lergue & Hérault & Hérault & 27 & 23 & 7 \\
\hline fev. 92 & Lolau & Lergue & Hérault & Hérault & 25 & 23 & 7 \\
\hline fev. 92 & St Fréchoux & Lergue & Hérault & Hérault & 40 & 33 & 7 \\
\hline fev. 92 & Cartels & Lergue & Hérault & Hérault & 28 & 93 & 7 \\
\hline fev. 92 & Capitoul & Lergue & Hérault & Hérault & 29 & 93 & 7 \\
\hline fev. 92 & Maubrun & Lergue & Hérault & Hérault & 30 & 100 & 7 \\
\hline fev. 92 & Poujols & Lergue & Hérault & Hérault & 17 & 100 & 7 \\
\hline
\end{tabular}




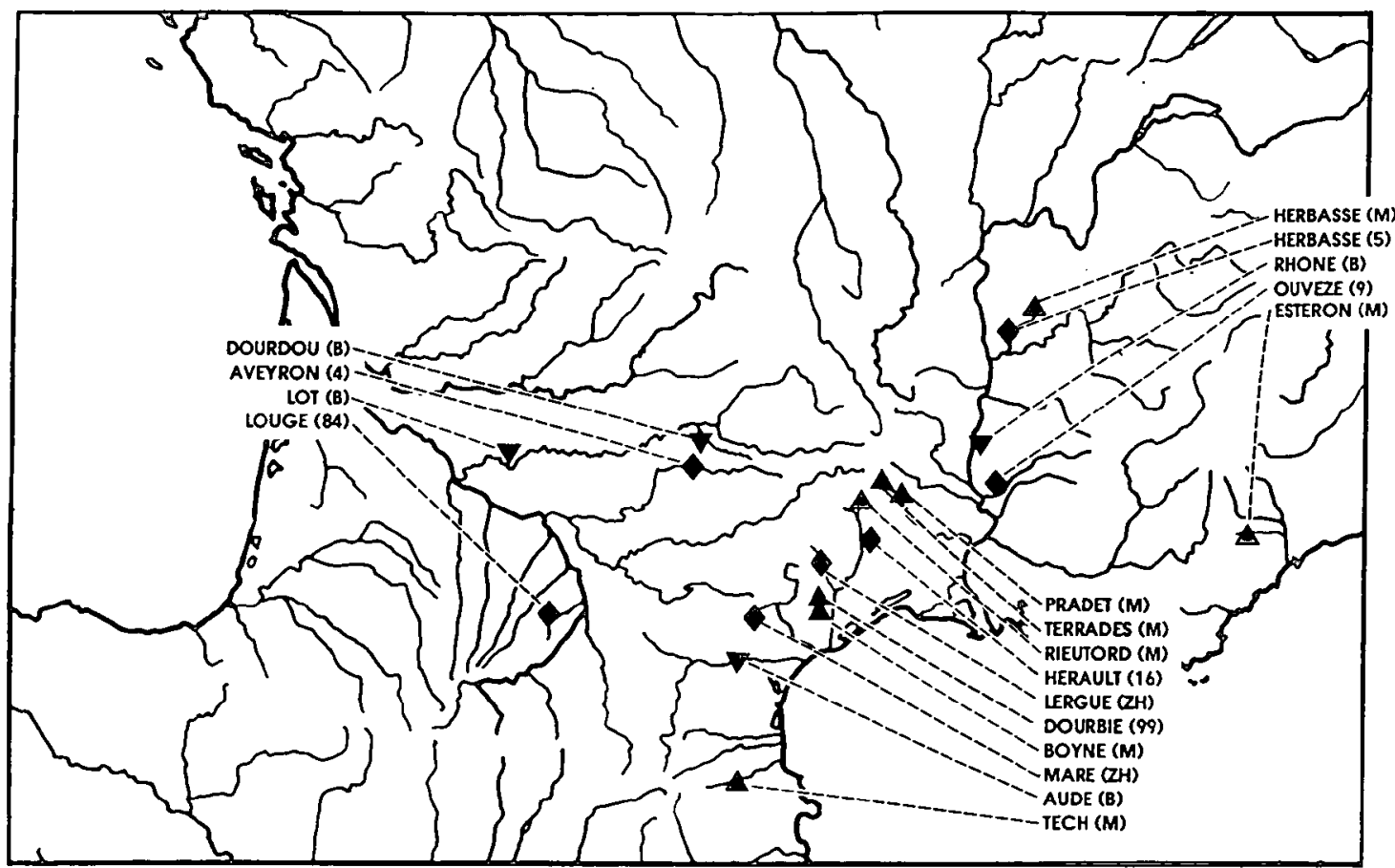

Figure 2 : Carte d'introgression en France : à la suite des noms des principales stations d'échantillonnage, $(M)$ correspond à $B$. meridionalis pur ; $(B)$ à des $B$. barbus purs ; les nombres représentent le pourcentage de gènes de $B$. meridionalis dans l'échantillon hybride (= GI, voir texte) ; $(\mathrm{ZH})$ sont des zones hybrides avec un cline d'introgression (voir tableau I).

Figure 2 : Map of barbel introgression in France : after the names of the main stations, $(M)$ corresponds to pure $B$. meridionalis populations ; $(B)$ to pure $B$. barbus ; the numbers represent the percentage of $B$. meridionalis genes in hybrid samples ( $=$ Gl, see text) ; $(\mathrm{ZH})$ are hybrid zones with clines of introgression (see table I).

- d'autre part, un affluent de la Garonne, la Louge, a été clandestinement empoissonné avec des barbeaux méridionaux provenant des Pyrénées-Orientales. Une analyse ponctuelle en 1986 a montré que l'introgression à la station «le Touch» atteignait $84 \%$. Un nouvel échantillonnage, en cours d'analyse, en trois points de la Louge contrôlera ce résultat surprenant et rendra compte de la structure de l'hybridation.

\section{L'HYBRIDATION}

\section{Analyse génétique du transect}

Dans une rivière du Sud de la France (la Lergue, tributaire de l'Hérault, près de Montpellier), des clines enzymatiques ont été mis en évidence entre les deux espèces de barbeaux en 1988 (Figure 3). L'analyse génétique porte sur 10 locus enzymatiques diagnostiques révélés par électrophorèse sur gel d'amidon (BERREBI et al., 1993). Un deuxième transect à mailles plus fines effectué en 1992 (CRESPIN, 1992) a permis la confirmation de ces clines et l'analyse génétique plus précise de la structuration des populations de barbeaux. Signalons qu'aucun génotype hybride F1 (c'est-à-dire hétérozygote à tous les locus diagnostiques) n'a été rencontré dans la Lergue lors de ces analyses. 


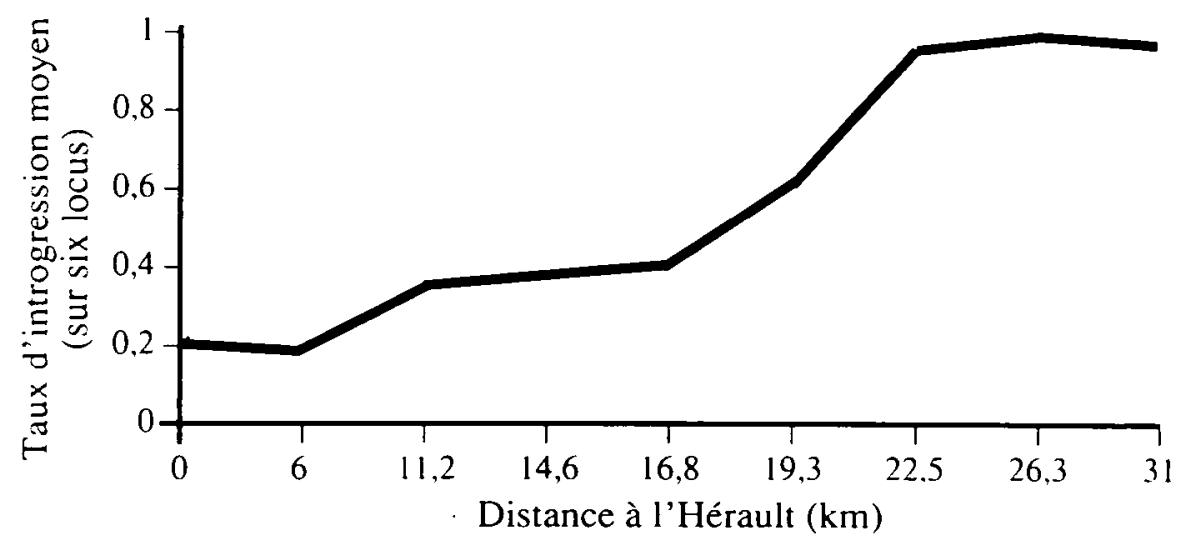

Figure 3 : Taux moyen d'introgression (GI, voir texte) en fonction de la distance à la confluence avec l'Hérault.

Figure 3 : Mean introgression rate (GI, see text) related to the distance of the station to the Herault river confluence.

\section{Zone de tension}

Ces clines enzymatiques démontrent que nous sommes en présence d'une zone hybride, c'est-à-dire d'une hybridation génétiquement structurée dans l'espace, avec une localisation réduite de l'hybridation.

D'après nos données actuelles, d'ordre essentiellement génétique, cette zone hybride semble bien correspondre au modèle "zone de tension". La théorie des zones de tension (BARTON et HEWITT, 1983 et 1985) postule que la zone hybride est le résultat d'un équilibre dynamique entre la dispersion des individus hybrides et leur contre-sélection. Une des prédictions de cette théorie est un déséquilibre de liaison fort (déséquilibre affectant la présence simultanée d'allèles appartenant à des locus différents dans un même individu). Or, un tel déséquilibre a été constaté dans cette zone hybride (CRESPIN, 1992). II serait créé par l'apport de génotypes migrants, provenant de populations aux fréquences alléliques différentes (de l'aval ou/et de l'amont). Le cline des fréquences alléliques le long de la rivière serait la traduction d'une sélection naturelle dirigée contre les génotypes hybrides (BARTON et HEWITT, 1983 et 1985).

Des indices de cette contre-sélection des hybrides ont donc été recherchés. Une expérience menée au laboratoire (POUYAUD, 1990) a permis de détecter une surconsommation en oxygène chez quelques hybrides et non chez les espèces parentales.

D'autre part, une étude de l'asymétrie du corps des barbeaux le long de la zone hybride a été réalisée (DARIUS, 1993 ; DARIUS et BERREBI, 1993) sur plusieurs caractères morphologiques. L'asymétrie fluctuante ou aléatoire passe pour être indicatrice de l'homéostase de développement d'un organisme, donc indirectement de la valeur adaptative de cet organisme (VAN VALEN, 1962). Les hybrides, pour plusieurs caractères, ont été trouvés plus asymétriques que les deux espèces parentales. Ceci serait donc le signe d'une moins bonne homéostase de développement, donc d'une dysgénèse hybride.

Cette étude a aussi mis en relief l'existence d'anomalies morphologiques au niveau du dernier rayon dur de la nageoire dorsale des hybrides.

Ces deux études, quoique ne fournissant pas de preuves à proprement parler, sembleraient indiquer que la valeur sélective des hybrides est plus faible que celles des deux espèces parentales. 


\section{Hybridation expérimentale}

Des hybrides de première génération et des backcrosses ont été obtenus dès 1988 (PHILIPPART et BERREBI, 1990). Selon cette étude, ces hybrides F1 ont sensiblement le même taux de croissance et présentent le même dimorphisme sexuel que le barbeau commun durant les premiers 24 mois d'existence. On attendait plutôt des caractéristiques intermédiaires, cette anomalie est probablement due à un fort "effet maternel" (les femelles utilisées étaient des barbeaux communs). Les patrons génétiques hybrides obtenus par électrophorèse des allozymes sont par contre l'assemblage additif des deux patrons génétiques des deux espèces parentales.

L'information la plus intéressante du point de vue évolutif tirée de ces expériences est la stérilité des mâles F1. Ceci constitue indéniablement un indice de contre-sélection de ces hybrides, condition sine qua non de la théorie de la zone de tension.

\section{Ethologie}

POUYAUD (1990) a entrepris de caractériser le préférendum écologique de chacune des deux espèces dans la Lergue. Pour cela, il a capturé plus de 100 individus par pêche électrique, sur des stations différentes définies à partir de critères physiques. La comparaison des caractéristiques enzymatiques des barbeaux avec les caractéristiques écologiques des stations où chacun a été capturé a permis de montrer, selon l'auteur, que les hybrides majoritairement meridionalis (c'est-à-dire portant une majorité d'allèles enzymatiques de ce type) se trouvaient plutôt dans les endroits à lumière tamisée, à courant faible ou nul et à fond vaseux. Les hybrides majoritairement barbus se rencontraient dans les milieux ouverts et à courant fort.

Dans la même étude, POUYAUD (1990) a tenté de caractériser, en aquarium, le comportement des deux espèces vis-à-vis de deux facteurs du milieu (courant et lumière), avec des expériences de groupe et des expériences individuelles. Selon l'auteur, le rôle de ces facteurs dans le comportement de choix d'un microhabitat n'a pas pu être mis en évidence à cause du trop grand stress manifesté par les barbeaux.

Des études sur le comportement, pendant la parade nuptiale et la ponte, ont aussi été réalisées chez les deux espèces (JEANDARME, 1991 ; JEANDARME et al., 1992 ; PONCIN et al., 1994). Dans ces études, des lots hétérospécifiques et homospécifiques de cinq poissons ont été réalisés, toujours avec une femelle et quatre mâles. Ces auteurs ont noté la fréquence de huit types caractéristiques de comportements élémentaires déjà connus chez les barbeaux (GOUGNARD et al., 1987), avant, pendant et après la ponte. Ces travaux ont montré que les différences de comportement étaient minimes entre les deux espèces et qu'elles se reconnaissaient lors d'expériences à lots hétérospécifiques. Le paramètre éthologique ne semble donc pas jouer de rôle négatif lors de l'hybridation.

\section{Parasitologie}

Des études parasitologiques (LE BRUN, 1988, 1989 et 1990 ; LE BRUN et al., 1988 et 1992) ont permis de conforter le fait que les deux espèces occupent des habitats différents, dans la zone hybride. Un parasite monogène spécifique de Barbus meridionalis a été mis en évidence dans la Lergue, Diplozoon gracile. Le barbeau commun n'est jamais infesté par ce parasite in natura (LE BRUN et al., 1988). Par contre, l'infestation expérimentale de Barbus barbus par ce parasite est possible, montrant qu'il n'y a pas de barrière physiologique. Le fait que seul Barbus meridionalis soit infecté in natura est interprété comme la conséquence d'une barrière éthologique : une différence de préférendum d'habitat entre les deux taxons de barbeaux. Le stade infestant du parasite se tiendrait dans des habitats fréquentés seulement par le barbeau méridional (zones sombres et calmes). A l'appui de cette hypothèse, il faut signaler qu'un autre cyprin, le goujon, Gobio gobio, dont l'écologie est très proche de celle du barbeau méridional (selon LE BRUN et al., 1992) est également infesté par ce Monogène.

Dans la zone hybride de la Lergue, une corrélation significative a été mise en évidence entre la prévalence (proportion de poissons parasités) et l'indice génétique GI 
(taux d'introgression). Il est donc probable que, plus un hybride est génétiquement proche du barbeau méridional, plus son comportement se rapprochera de celui de cette espèce, plus il aura de chances d'entrer en contact avec le parasite spécifique et donc plus la probabilité qu'il soit parasité sera forte.

\section{DISCUSSION ET CONCLUSION}

\section{Historique de l'hybridation}

L'ensemble des données réunies dans ce texte nous permet de proposer un scénario possible de l'origine de l'hybridation entre les deux barbeaux français.

Tout d'abord, l'existence de cas d'hybridation dans des cours d'eau différents (Orb, Hérault, Rhône), les hybridations artificielles observées après transplantation (Garonne) ou obtenues expérimentalement (Belgique) nous permet de penser que ce n'est pas un phénomène nécessitant des conditions exceptionnelles, si ce n'est la rencontre des deux espèces.

Quand a eu lieu cette rencontre ? Nous savons que les peuplements de barbeaux méridionaux dans le Massif Central (et probablement des Pyrénées et des Alpes françaises) sont issus de recolonisations postglaciaires, il y a environ 10 à 15000 ans (PERSAT et BERREBI, 1990) ; ce qui explique le faible polymorphisme enzymatique observé, voire le monomorphisme total (BERREBI et al., 1988). Or, aucune trace de gènes de Barbus barbus n'a été décelée dans ces populations colonisatrices. On peut en déduire qu'il n'y avait pas d'hybridation avant cette recolonisation. Cette rencontre, sans influence humaine, a donc probablement eu lieu après les dernières glaciations (BERREBI, sous presse) du fait de l'arrivée probablement postglaciaire du barbeau commun sur les côtes méditerranéennes françaises. A l'appui de cette hypothèse, notons que, selon BARTON et HEWITT (1985), la plupart des zones hybrides datent de la fin des dernières glaciations.

On peut aussi penser que le croisement hétérospécifique, lors des premières années de mise en contact, s'est fait sans difficultés puisque l'étude éthologique de la reproduction a montré une presque parfaite concordance des deux espèces (PONCIN et al., 1994).

Actuellement, même si on ne peut dater avec certitude l'origine des différents cas d'hybridation, il est clair que les deux espèces pures ne se rencontrent plus directement, puisqu'aucun F1 n'a été observé : les populations hybrides naturelles sont en fait composées d'individus issus de croisements divers entre hybrides complexes. Toutes les combinaisons génétiques peuvent se rencontrer le long de la zone hybride, ce sont les proportions statistiques des gènes des deux espèces pures à chaque station analysée qui varient.

\section{Classification du modèle barbeau}

On peut tenter d'expliquer l'asymétrie de cette introgression par le fait que Barbus barbus est réellement désavantagé en altitude, tandis que $B$. meridionalis peut vivre en plaine s'il est libéré de la compétition vis-à-vis de l'autre barbeau. On peut aussi ajouter que la dévalaison passive des barbeaux (et donc de leurs gènes) est possible tandis que toute remontée doit être volontaire.

D'autre part, en plein centre de la zone hybride de la Lergue, POUYAUD (1990) a démontré que le microhabitat choisi par chaque hybride est en partie fonction de sa composition en gènes parentaux, c'est-à-dire au taux d'introgression. Les résultats des analyses parasitologiques aboutissent au même type de résultats. LE BRUN et al. (1992) montrent que si la présence du parasite Diplozoon gracile est fonction du taux d'introgression de l'hôte, ce n'est pas pour des raisons physiologiques mais probablement par un processus indirect : le taux d'introgression doit influer sur le comportement, qui lui même doit influer sur les possibilités de rencontre hôte-parasite.

Cette finesse de comportement liée à la composition génétique s'ajoute aux capacités de ces espèces à appréhender l'environnement habituel, probablement «au caillou près». On sait déjà que la capacité à choisir son habitat est très forte chez le barbeau commun (BARAS, 1993). 
Tous ces éléments nous permettent de proposer que le facteur comportemental (et en particulier la "psychologie" de ces poissons les poussant à se maintenir dans un environnement réduit qu'ils connaissent bien) est une composante essentielle de cette zone hybride d'un type original.

Actuellement, peu de données écologiques sur le déplacement des individus sont disponibles, la question reste donc ouverte. Une étude de marquage et de capture/recapture qui a débuté en 1993 devrait permettre de connaître précisément les mouvements des barbeaux en zone hybride.

\section{Avenir des hybridations}

Dans le genre Barbus, d'autres cas d'hybridation sont connus.

B. meridionalis s'hybride avec B. haasi en Catalogne (MACHORDOM et al., 1990). Cette hybridation concerne un seul petit bassin : le Besos, où une migration a mis en présence respectivement $89 \%$ et $11 \%$ de chaque espèce. L'échantillon du Besos est donc considéré comme un pool génétique ayant subi des sélections importantes depuis sa constitution.

B. meridionalis (ss. sp. petenyi) s'hybride avec $B$. barbus en Slovaquie orientale (SLECHTOVA et al., 1993). Contrairement au cas français, la zone de sympatrie des deux espèces le long du cours d'eau est très importante, avec une faible polarité altitudinale. Malgré cette promiscuité, on trouve peu d'individus hybrides (entre 0 et $9 \%$ ). Une barrière à définir limite donc fortement l'introgression.

L'ensemble de ces données nous montre que, pour chaque cas, un processus différent semble empêcher une homogénéisation totale des espèces : arrêt du flux migratoire en Espagne, une barrière à la reproduction encore inconnue en Slovaquie qui limite le nombre d'hybrides, pourtant fertiles (SLECHTOVA et al., 1993), zone de tension en France. Ce constat est logique car si aucune barrière ne s'opposait à l'homogénéisation des deux partenaires d'une hybridation, une troisième espèce synthétique serait apparue, ce qui est peut-être l'avenir du cas espagnol. De ces trois processus, celui de la zone de tension est le plus énigmatique. Bien qu'analysé depuis de nombreuses années de façon interdisciplinaire, il est encore difficile d'en prévoir l'évolution. L'existence d'une introgression d'environ $15 \%$, généralisée à tout l'Hérault, et l'extension de cette hybridation à tous les bassins de sympatrie des deux espèces, suggèrent cependant un processus qui aboutirait progressivement à une modification de $B$. barbus, en l'enrichissant d'allèles compatibles d'origine meridionalis. La zone de tension servirait alors de barrière, ne permettant qu'à des combinaisons neutres ou avantageuses de passer en plaine et protégeant l'intégrité de $B$. meridionalis.

\section{Intérêt du modèle Barbus}

Le modèle de l'hybridation entre Barbus barbus et Barbus meridionalis a suscité de nombreuses recherches dans des domaines scientifiques fort variés. Les collaborations nombreuses que ce sujet a favorisées ont permis d'en envisager l'étude par une approche multidisciplinaire. Chaque apport d'une nouvelle discipline a contribué à l'enrichissement des connaissances déjà acquises, en suscitant de nouvelles hypothèses et/ou en infirmant ou confirmant des hypothèses établies. L'étude portant sur la théorie des zones hybrides peut être avantagée grâce à certaines simplifications permises par le modèle Barbus. En effet, le système considéré est linéaire (la rivière), ce qui réduit l'espace à une dimension. La comparaison possible entre plusieurs hybridations voire zones hybrides d'âges vraisemblablement différents (ce qui constitue une opportunité rare) devrait permettre de tester la dynamique du phénomène, ce qui peut être une des façons de tester les différents modèles proposés dans la littérature. Des caractéristiques qui tiennent directement au matériel biologique considéré sont également des données-clés. Les populations de barbeaux sont de taille relativement réduite et leur capturabilité est forte. Le modèle Barbus apparaît donc comme un modèle privilégié pour départager les différentes hypothèses de la théorie des zones hybrides.

L'aspect multidisciplinaire de ce thème de recherche apparaît donc comme une richesse et c'est cet aspect (essentiel !) qui a permis d'arriver aussi rapidement à une telle 
somme de connaissances. Il n'en demeure pas moins que bien des inconnues restent posées. Peut-être de nouvelles disciplines se feront-elles fort de les résoudre dans les années à venir...

\section{L'AVENIR}

L'essentiel de nos travaux à Montpellier va porter sur l'étude plus fine, des points de vue génétique, morphologique et dynamique de la zone hybride de la Lergue et sur quelques aspects écologiques de cette hybridation particulière. Des études plus ponctuelles auront lieu sur les autres hybridations connues pour établir des comparaisons afin de mieux comprendre la mise en place et le fonctionnement des hybridations et des zones hybrides.

Nous allons aborder, dans la zone hybride de la Lergue, l'étude des mouvements des animaux avec la mise en oeuvre d'une étude de marquage et de capture/recapture des barbeaux le long de la zone hybride. Ce travail a déjà commencé et a pour but d'évaluer les capacités du poisson à la migration efficace, paramètre déterminant pour le modèle génétique de la zone de tension. Nous disposons de marques permettant d'identifier chaque individu («P.I.T.-Tags"): Des marqueurs génétiques fiables permettent l'approche génétique du problème. Il est donc envisageable d'arriver à caractériser précisément les relations tant génétiques que "somatiques" entre populations dans un futur relativement proche. Les résultats diront si le ratio entre la migration et la sélection (seulement perceptible au niveau génétique) suffit à rendre compte des caractéristiques observées (théorie de la zone de tension) ou si seule l'amplitude de la migration est à prendre en compte (supériorité hybride, modèle neutre).

Nous envisageons maintenant de passer aussi à un autre niveau d'analyse génétique en ayant recours aux marqueurs de l'ADN, qu'il soit cytoplasmique ou nucléaire. Une étude de RFLP sur l'ADN mitochondrial est déjà en cours. Elle devrait permettre de préciser le sens dans lequel s'est fait le croisement interspécifique. Logiquement, vu le dimorphisme sexuel net en faveur des femelles et le déroulement de la reproduction chez les deux espèces (HANCOCK et al., 1976). on peut s'attendre à ce que ce soient surtout des mâles de barbeaux méridional qui se sont croisés avec des femelles de barbeaux communs. II sera aussi intéressant de comparer les deux clines alors à notre disposition. On sait que chez de nombreuses espèces (les deux sous-espèces de souris domestique d'Europe par exemple) les deux clines ont des profils assez, voire totalement différents dans la zone hybride (VANLERBERGHE, 1988). La maîtrise des microsatellites, obtenus à partir de l'ADN nucléaire, prévue à l'horizon de 1995, éclaircira, quant à elle, les relations de parenté entre les individus d'une même station. D'autres retombées peuvent également se produire, notamment en ce qui concerne la comparaison avec les clines allozymiques.

\section{REMERCIEMENTS}

Les auteurs remercient de leur aide indispensable tous les chercheurs cités en bibliographie ainsi que P. LIM et Y. SOUCHON. Ils remercient également la Fédération des AAPP de l'Hérault, la 8ème Délégation Régionale du Conseil Supérieur de la Pêche, la Communauté Européenne et les membres du Laboratoire de Parasitologie Comparée de Montpellier pour l'aide efficace qu'ils ont apportée durant plusieurs années.

\section{BIBLIOGRAPHIE}

BARAS E., 1993. Etude des stratégies d'occupation du temps et de l'espace chez le barbeau fluviatile, Barbus barbus (L.). Cah. Eth. appl., 12 (2-3), 125-442.

BARTON N.H., HEWITT G.M., 1983. Hybrid Zones as barriers to genes flow in Systematics Association Special Vol. 24, “Protein Polymorphism : Adaptative and Taxonomic Significance», G.S. Oxford and D. Rollinson (Eds.), Academic Press, London and New York.

BARTON N.H., HEWITT G.M., 1985. Analysis of hybrid zones. Ann. Rev. Ecol. Syst., 16, 113-148. 
BERREBI P., 1984. Génétique enzymatique du barbeau méridional (Barbus meridionalis) dans le Parc des Cévennes. Grant rapport. Univ. Montpellier II. 38 p.

BERREBI P., 1991. High frequencies of null alleles and functional diploidization in tetraploid Barbus (stricto sensu) (Osteichtyes, Cyprinidae). Poster, Third Congress ESEB, Debrecen, Hungary.

BERREBI P., 1994 (sous presse). Speciation of the genus Barbus in the North Mediterranean basin : recent advances from biochemical genetics. Biological Conservation.

BERREBI P. (en prép.). The genetic structure of the hybrid zone between the barbels Barbus barbus and $B$. meridionalis (Osteichtyes, Cyprinidae) : importance of the behavioural factor.

BERREBI P., LE BRUN N., RENAUD F., LAMBERT A., 1987. Hybridation Interspécifique de deux Cyprinidae (Genre Barbus). Conséquences sur la spécificité parasitaire de Diplozoon gracile (Monogenea). Coll. Nat. CNRS «Biologie des Populations", 179-180.

BERREBI P., LAMY G., CATTANEO-BERREBI G., RENNO J.F., 1988. Variabilité génétique de Barbus meridionalis Risso (Cyprinidae) : une espèce tétraploïde quasi monomorphe. Bull. Fr. Pêche Piscic., 310, 77-84.

BERREBI P., DUPONT F., CRIVELLI A.J., 1989. An isozyme study of the natural cyprinid hybrid Alburnus alburnus x Rutilus rubilio in Greece. J. Fish Biol., 34, 307-313.

BERREBI P., CATTANEO-BERREBI G., LE BRUN N., 1993. Natural hybridization of two tetraploid barbels: Barbus barbus and $B$. meridionalis (Pisces, Cyprinidae) of southern France : morphology and genetics. Biol. J. of the Linn. Soc., 48, 319-333.

BLONDEL J., 1986. Biogéographie évolutive. Coll. d'écologie. Ed. Masson, Paris. 221 p.

CAMPTON D.E., 1987. Natural hybridization and introgression in fishes : methods of detection and genetic interpretations in N. Ryman and F. Utter (eds.), Population genetics and fisheries management, 161-192. Seattle : University of Washington Press.

CAMPTON D.E., 1990. Application of biochemical and molecular markers to analysis of hybridization in D. H. Whitmore (ed.), Electrophoretic and Isoelectric focusing techniques in fisheries management, 241-264. Boca Raton, Floride : CRC Press.

CRESPIN L., 1992. Structure génétique de la zone hybride Barbus barbus $\times$ B. meridionalis (Cyprinidae) de la Lergue (Hérault). D.E.A., Univ. Montpellier II, 20 p.

DARIUS H., 1993. Etude de l'asymétrie de Barbus barbus et Barbus meridionalis (Cyprinidae) dans la zone hybride de la Lergue (Hérault). Rapp. Maîtrise, Univ. Montpellier II, 19 p.

DARIUS H., BERREBI P., 1993. Asymétrie et anomalies morphologiques dans la zone hybride de la Lergue (sud de la France) : Barbus barbus x Barbus meridionalis. Cah. Eth. appl., 13 (2), 243-244.

ECONOMIDIS P.S., SINIS A.J., 1988. A natural hybrid of Leuciscus cephalus $x$ Chalcalburnus chalcoides macedonius (Pisces, Cyprinidae) from Lake Volvi (Macedonia, Greece). J. Fish Biol., 32, 593-605.

GOUGNARD I., PONCIN P., RUWET J.C., PHILIPPART J.C., 1987. Description et analyse du comportement de reproduction du Barbeau Barbus barbus (L.) en aquarium. Influence du nombre de mâles courtisants sur les comportements observés. Cah. Eth. appl., 7 (3), 293-302.

HAFEZ R., 1981. Etudes cytogénétiques et évolution génétique chez quelques Cyprinidés de la région Midi-Pyrénées. Thèse Inst. Nat. Polytechnique Toulouse, $362 \mathrm{p}$.

HANCOCK R.S., JONES J.W., SHAW R., 1976. A preliminary report on the spawning behaviour and nature of the sexual selection in the barbel, Barbus barbus (L.). J. Fish Biol., 9, 535-542. 
JEANDARME J., 1991. Etude préliminaire du comportement d'hybridation de Barbus barbus (L.) et Barbus meridionalis (R.) en aquarium. Mémoire de Licence. Univ. de Liège. $55 \mathrm{p}$.

JEANDARME J., PONCIN P., BERREBI P., 1992. Etude préliminaire du comportement d'hybridation de Barbus barbus (L.) et de Barbus meridionalis (Risso) en aquarium. Cah. Eth. appl., 12(4), 519-528.

KONKLE B.R., PHILIPP D.P., 1992. Asymmetric hybridization between two species of sunfishes (Lepomis : Centrarchidae). Mol. Ecol., 1, 215-222.

LE BRUN N., 1988. Spécificité des relations hôtes-parasites : le modèle CyprinidaeDiplozoon. Thèse Univ. Montpellier II, $168 \mathrm{p}$.

LE BRUN N., 1989. Spécificité des relations hôtes-parasites : le modèle CyprinidaeDiplozoon. Bull. Ecol., 20, 86-88.

LE BRUN N., 1990. Differential settlement by Diplozoon gracile (Monogenea) on teleostean (cyprinid) hosts in relation to biological and environmental conditions. Acta Oecologica, 11, 729-739.

LE BRUN N., RENAUD F., LAMBERT A., 1988. The genus Diplozoon (Monogenea, Polyopistocotylea) in Southern France : speciation and specificity. Int. J. Parasitology, 18 (3), 395-400.

LE BRUN N., RENAUD F., BERREBI P., LAMBERT A., 1992. Hybrid zone and host-parasite relationships : Effect on the evolution of parasitic specificity. Evolution, 46 (1), 56-61.

MACHORDOM A., BERREBI P., DOADRIO I., 1990. Spanish barbel hybridization detected using enzymatic markers : Barbus meridionalis Risso x $B$. haasi Mertens (Osteichtyes, Cyprinidae). Aquat. Living Res., 3 (4), 295-303.

MENZEL B.W., 1976. Biochemical systematics and evolutionary genetics of the common shiner species group. Biochem. Syst. Ecol., 4, 281-293.

PERSAT H., BERREBI P., 1990. Relative ages of present populations of Barbus barbus and B. meridionalis (Cyprinidae) in Southern France : preliminary considerations. Aquat. Living Res., 3 (4), 253-263.

PHILIPPART J.C., BERREBI P., 1990. Experimental hybridization of Barbus barbus and Barbus meridionalis : physiological, morphological and genetic aspects. Aquat. Living Res., 3 (4), 325-332.

PONCIN P., JEANDARME J., BERREBI P., 1994. A behavioural study of hybridization between Barbus barbus and Barbus meridionalis. J. Fish Biol., 45, 447-451.

POUYAUD L., 1990. Ethologie, écologie et génétique de Barbus barbus, $B$. meridionalis et leurs hybrides sur la Lergue, affluent de l'Hérault. D.E.A. Univ. Montpellier II, $39 \mathrm{p}$.

SLECHTOVA V., SLECHTA V., BERREBI P., 1993. Diversité génétique du genre Barbus dans les rivières tchèques et slovaques. Cah. Eth. appl., 13 (2), 207-210.

VAN VALEN L., 1962. A study of fluctuating asymmetry. Evolution, 16, 125-142.

VANLERBERGHE F., 1988. Histoire évolutive de la zone d'hybridation naturelle entre deux sous-espèces de souris européennes: Mus musculus domesticus et Mus musculus musculus. Dynamique de l'introgression des gènes autosomaux, de l'ADN mitochondrial et du chromosome $Y$. Thèse Univ. de Montpellier. $115 \mathrm{p}$.

VERSPOOR E., HAMMAR J., 1991. Introgressive hybridization in fish : the biochemical evidence. J. Fish Biol., 39 (supplement A), 309-334. 